\title{
Haptic Interactions through Touch-Screen Interfaces
}

\author{
Irida Ntalla \\ City University \\ London \\ UK \\ irida.Ntalla.1@city.ac.uk
}

\begin{abstract}
In this paper 'touch' is discussed in relation to the questionable claims of interactivity within museum and gallery spaces. I examine audiences' experiences when using touch sensitive interfaces and bodily contact with digital environments. With a tap, a slight slide or a soft hand touch interfaces are engaged with and allow access to a range of information, content and experience. The audience's engagement with these types of digital 'interactives' seems to aspire to provide emotional, affective and personal experiences. The discussion will include an exposition of how audiences respond on their experience in relation to interactivity and touch within the museum environment. The findings from a qualitative research project conducted in the Modern Galleries of the Museum of London exposed touch as an important characteristic of these experiences.
\end{abstract}

Touch-screen interfaces. Haptic. Interactivity. Audience experience. Play.

\section{TOUCH IN THE MUSEUM}

The museum finds itself in continuous negotiation of its identity and role to engage in long standing debates about its existence. This complex situation fights negative connotations of the museum such as the unpleasant overtones of 'museal' (Adorno 1983) or 'mediative necropolis' giving us a thieves' conscience with the exhibits ending up hung on those walls for the appreciation of Sunday visitors or Monday intellectuals (Merleau-Ponty 1998). The noble character that the museum imposes when entering its space demands respect and most often silent admiration towards the artefacts. The relationship between the audience and exhibits is visual and in a physically motionless state. The collection and showcase of these objects that convey the guardianship of authenticity is a historical mission. The need and necessity for a philosophical re-invention of the museum is an active discussion within museum studies debates.

Hein (2000) attempts to clarify the role of objects within museum spaces and practices today, reasoning that objects reconstitute sites of experience. Indeed, current understandings of the museum are widely expressed as a shift in focus away from the object towards spaces of cultural interactivity (Martin et al. 2007). Interactivity comes into play as a 'tool' and a form that promise experiences with interaction bringing the visitors in action, movements, personal and sensory participation and engagement. The inherent subjectivity of experience endangers the museum's claim to authority over its representation (Hein 2000). Museums are engaged in an entirely new undertaking with a focus on experiences, provoking thoughts and emotions.

The embracement of technological innovation offers great promises on the democratization of knowledge, the contextualization of information on exhibits and the prospect to boost museum attendance (Griffiths 2009). Interactive and new technologies are also associated with the empowerment of the museum visitors, by providing them choices through experience allowing space for many voices. The experience when engaging with an artefact, object, digital device or digital mediated environment moves beyond a cognitive exchange, beyond intellectual engagement and towards a felt one.

The idea of touch is arousing as it allows the activation of sensory engagement moving the audience-exhibit relationship away from the pure visual one. Touch in museums, initiated through the allowance of touching museum objects making available primary objects or copies of them to audiences arguing the enhancement of sensory experience through the proximity with the objects (Cranny-Francis 2007). Other examples of practices related to touch that act within and outside the museum walls incorporate educational 
interventions where mainly children are given the opportunity to manipulate and touch specific objects of the museum, for the most part replicas, open treasure drawers, use of tangible tabletops and mechanical interactives. From the late 1960's with the ideas of Frank Oppenheimer and the development of the Exploratorium, challenged the element of pure visual perception and the strictness towards touching artefacts and exhibits, through the development of hands-on exhibits (Latham \& Wood 2012). These exhibits, significantly popular in science and children's museums are centring on educational and teaching purposes. It is interesting to note that due to the mentioned developments regarding the relationships with the hands-on exhibitions and handheld objects, the conception of touch and physical engagement in the museum encounters appears to be strongly associated with children and younger audiences, education and play.

Technologies have been approached by a number of research studies as the key answer to remodel these relationships and enhance the audience experience (Vom Lehn \& Heath 2005). Touch comes to play offering an immediacy and directness to these experiences particularly with the embracement of digital technologies. Zimmer and Jefferies (2007) quote interestingly Madaline Diacon and her questioning of what would be apart from the aspects of preservation that makes the museum so keen on using restrictions such as 'Do Not Touch'. She suggests knowledge that is waiting to be awakened by our fingerprints.

\section{TOUCH AND LEARNING}

Touching in the museum is argued to be essential to the museum experience by a number of thinkers and professionals, (Classen \& Howes 2006) still most explored from the aspects of learning. The idea of learning by doing and by being actively involved in the process is widely accepted and reinforces the importance of those exhibits. Different studies suggest that multisensory experiences of interacting with objects promote engagement, understanding and learning (Falk \& Storksdieck 2005).

Hein (1998) proposes that learning experience and knowledge acquisition requires active participation through both hands and minds. Activeness embedded in the meaning of play can occur most often through touching. In the museum context, play is mainly associated with the educational aspect directed to children separated from the overall museum environment and exhibitions. Historically, learning also has strong links with the conception of play. Numerous education theorists have long expressed the interrelations of construction of knowledge, learning, action and social interaction. According to Vygotsky (1978) social interaction and play are crucial for the cognitive development especially for children as they become active participants when they engage in problem-solving activities with their guides or peers. Social interaction is essential for learning to occur. Playfulness can exist in a number of interactions within the museum spaces and it should be integrated throughout the overall experience instead of being approached and practiced as an individual aspect. Elements such as play and touch have a long tradition of being viewed as add-ons to the exhibition experience lacking contextualization with the exhibits, acting as a diversion.

The shift from the visual relationship with the objects to a tactile and multisensory one, the appreciation of the role of touch within the museum space has blossomed. Touch is distinguished from the other senses such as vision, the main sense that has been the established mode that technologies have been delivered to us and we have focused on mostly until recently. This differentiation is argued to arise from the element of immediacy due to intense sensations, affect, pleasure or pain that is added to our engagement with technology, with the experience manifested to more part of the real world (Zimmer \& Jefferies 2007).

\section{TOUCH AND SENSES}

The human sense of touch, also known as 'haptic', a manifold and tangled conception and its existence as an individual sense have been argued since the period of Classical Greek philosophy. According to Aristotle touch is acknowledged as primary to the other sensory modalities, without it no other sense is possible. Flesh is the medium of the touch. If we place the object on the organ it is not perceived, if we place it on the flesh it is perceived; "therefore flesh is not the organ but the medium of touch (Paterson 2007: 15). Other thinkers, such as St Aquinas and up to Descartes have explored the sense of touch, as a tool for our body that without we may have no consciousness and know nothing. For St Aquinas it is actually presented as a first sense, "the first sense, the root and ground, as it were, of the other senses" (Aquinas, as cited in Jutte 2008). In the physiological work related to senses and consequently touch, David Katz (1925) a pioneer in this field declines the classification of lower and upper senses.

The sense of touch is being explored by phenomenological thinking, in large as insuring immediacy for the sense experiences from thinkers 
such Merleau-Ponty, Heidegger and Husserl (Elo 2012). Heidegger (1962) defines this as the 'science of phenomena', with the purpose to investigate and describe individual lived experiences. Touch is certainly more demanding than exploring it just through the lens of physical contact between the skins of bodies, or the skin and other materials and objects. Only considering the ways we use only linguistically the word touch in our everyday verbal and written encounters, it will allow us to apprehend its complexity. We touch, grasp, tap, press, contact, pat, strike, handle, feel not only physical objects but more than often immaterial existences related to space, concepts, ideas (e.g. 'She seems to have lost her touch!', 'this issue touches us all', 'I was really touched by his move', 'keep in touch' ).

To touch and be touched, to affect and be affected "is to bring aspects and forces of the world nearer to us" (Paterson 2007). The complexity of touch is noted by a long list of thinkers bringing it certainly close to various aspects of the human experience with an evident influence on the way we move in the spaces, affect and get affected by our surroundings. It is our bodies, specifically our sensorimotor apparatus that enables us to perceive, move and manipulate. The body is a site of perception, a field of actions. The human body allows a range of interactions, perceptions and noesis deriving or engaging touch that travel beyond its literal meaning enhancing its complex process. According to Deleuze "the conditions of experience in general must become conditions of real experience; in this case the work of art (museum) would really appear as experimentation (1990: 260).

\section{TECHNOLOGY AND TOUCH}

The philosophical and phenomenological perspectives have been increasingly prominent and attractive to fields such as computing, interaction design and human interaction design 'forced' by the realization of the users and their bodies as active agents that act, influence and engage in the environments they are (Dreyfus 1992, Dourish 2001). The demand of interactivity within culture and art has awakened the multisensory modes of perception challenging the traditional epistemological models that focus on visual perception and inner imaginative processes. This brings to light the entanglement between philosophy of reason, mind and body towards digital computation, a relationship that defines how we perceive and interact with computing. A critical look at the integration of the body, more specifically of tactility and movement, provides insights on the current state of tangible computing system, which refers to any computing system that integrates and emphasizes the role of the body and touch as part of the interaction. In the context of the humancomputer interface, touch ranks third in the hierarchy of the senses (Paterson 2007: 128).

Technology and digitality, the condition of living in a digital culture are closely entangled. The digital seems to unfold genealogically from differential relationships between the physical and the technological (Muster 2006: 256). Digital technologies and its application seem to offer the excitement to bring its content to our fingertips and according to Mika Elo (2012) the finger has moved to the status of a switch, dragging the whole body along. The act of the fingers, and therefore the hands brings the idea of poiesis, of doing, of influencing, of interactivity as any exchange of a subject with an object and it is up to the individual's or group's willingness and capacity to stimulate mental activity through this communication.

Touch as encountered through mediated environments and social technologies opens up further questions, due to technical and technological conditions, elements of distance and proximity, the familiar and affective factors of technics in the construction of experience. We use touch in our everyday actions and we had a certain 'comfort' and understanding on apprehending, interpreting and experiencing ways of touching the hot cup of coffee, a stranger in the streets, a pet etc. We have the capacity to understand when a pet enjoys the touching or a nervous tap on the desk. The connection when we touch a machine can carry a similar plot, challenging the human/machine binary of western thinking.

\subsection{Touch 'interactives' in the museum}

Museums have been responding to the advent and progress of the information age, by acquainting new methods of exhibition design, incorporating a range of technological exhibits that mainly aim to promote active participation through interactivity and immersion of the visitors as well as enhancing learning and increasing educational promises. In the last decade, more advanced forms of mixed interactive systems have been developed which include augmented reality (AR), mixed reality (MR), tangible and multimodal user interfaces (TUI) or 'tangible interactives' (Kidd et al. 2011). These latter terms recognize tangibility and materiality in new ways; embracing the physical embodiment of data, bodily interaction and embeddedness in real spaces and contexts (Hornecker 2009).

Interactives along with the multimedia kiosks are often understood as a way of providing a range of information and data, possibly as an aid for the audience to enhance the rest of the exhibitions or exhibits in contrast to the real and virtual objects 
that are seen to successfully reduce the distance between past and present objects (Pallud 2009). In a previous study (Kidd et al. 2012) it has been found that multisensory interactive installations increased visitors' interest and excitement when they entered the environment. However, following Hornecker's argument the idea of tangible interaction focuses on human control, creativity and social action rather than the representation and transmission of data - a shift, which would have significant implications for the role and status of interactives in museum spaces (Hornecker 2009).

The majority of research conducted by art and humanities disciplines in relation to conception touch within galleries and museum environment explore touch in relationship to real objects with a later expansion and interest on virtual artefacts and virtual touch. Virtual artefacts perform as substitutes of the real objects allowing audiences to touch and manipulate them. A number of works have been conducted on the $3 D$ virtual environments and the characteristics of successful haptic systems for the museum exhibits and objects or the 'feelable details' that is required. These inquiries are included also in a current discussion generated by Bentkowska-Kafel et al. (2012) that incorporate discussions on effective and realistic user experience that can emerge from the use of haptic interfaces and technological limitations of the current haptic interfaces. Additionally, looking into the learning benefits of the use of virtual objects is another popular and extended area of research. Touch and haptic have been explored extensively in relation to artworks bringing in notions such as haptic aesthetics.

\subsection{Touch, haptics, interactivity}

This area of technology known as 'haptics' develop environments and devices that involve the sense of touch or tactile sensations (Paterson 2007) as well as vision and sound, viewed as multi-sensory. The term haptic comes from the Greek work haptikos that means 'able to come in contact with', a concept of defining the science of human touch (Jutte 2008). Frequently, notions such as tactile, tactual or haptic are apprehended as synonymous with touch. However the notion of tactile relatepurely to the literally whereas haptic declines the contrast between vision and touch and as Deleuze \& Guattari (1987: 479) argue the haptic can be as much visual or auditory as tactile. They identify the haptic (from Greek ämTw, able to come in contact) with their notion of smooth space: a space of affects, navigated by constant reference, by constantly acting on feedback from an immediate environment. The "smooth space is occupied by intensities, wind and noise, forces, and sonorous and tactile qualities" (p. 480) with locations and relations. The haptic is not about the tactile more than it is about visual or auditory sensation, it is about participation. Haptic interfaces argue to allow the audience to become active agents through the interaction with technology and the body is opened a new type of touch to itself as well as objects and other bodies (Elo 2012).

This active and dynamic type of touch does not oppose eyes with hands declaring a common sensory existence and interdependence where when your hands come into a contact with a surface, equally your vision engages with the movement of your body, the image, the colours, the graphics that the surface is enveloped with. The concept of haptic demands the incorporations of space and time involving proximity and distance within it. Following the deleuzian view to the visual and tactile understanding of haptic, this notion emerges for them in the 'smooth space', a space of affects, haptic rather than visual where events occur. Haptic related to movement, activeness and perception of stimuli leads towards the phenomenological understanding of embodied experiences. Gibson's (1966) definition of haptic as touch and vision, an apparatus that allows the communication of the human body with the information available, limits the potential of haptic systems to an interaction with existing facts and given data and the body to a receiving machine.

Interactivity seeks audiences to affect and be affected (Deleuze 1987) through various rhythms and modalities of encounter and channels of sensation and sensibility leading to becoming. Interactivity in the context of the museum is been translated on a great degree with the celebrated phrase 'Please touch!' Digital technologies offer the potential to manipulate and intervene in media referred as the interactive potential of new media (Lister et al. 2003). Touch through technologies such as digital and interactive media is mediated with the medium acting as an enabler to connect things on the one side but also as something that palpably stands between (Zimmer \& Jefferies 2007) proximity. The common argument on the differentiation of touch from the other senses is the proximity of the two entities, touched and being touched in a distance where no space, part the air that floats around, remains between them.

The interactives and interactivity in the museum has brought the conception of touch to a different level where with just a slight tap the user is allowed to access information, discover worlds of past and future, come close to other people and histories. Interactivity and touch have come to a very close relation and it does require closer investigation. The modern museum environments certainly more technologically friendly are embracing enthusiastically interfaces with finger-friendly touch points. 


\subsection{Touch-screen interfaces in the Museum of London: Emergent findings on the nature of interaction, touch and affect}

Haptic technologies are increasingly popular in high-end workstations and manufacturers, as well as lower-end home computers, phones etc. Our everyday life is excessively being dressed with devices that require our fingers to be manipulated. These types of displays are seeking our skilful finger slighting to come alive and its use manifests itself through every moment of the daily life. The design of the desktop computer requires us to type at the keyboard and click the buttons of a computer mouse, the designs of mobile phones and portable music players require us to push buttons or stroke a touchpad, etc. The increasing employment of touch in the user interfaces in everyday and popular consumer electronics surely had its impact as mentioned above on the choice of design and display of exhibition in the museum as well as on the way the audiences relate with the exhibit, their expectations and experience in the museum space.

Popular electronic devices such as Apple's iPod music player and iPad have certainly triggered a revolution in our relation and familiarity with the touch sensitive screens. These interfaces also integrate the body and a broad set of tactual actions offering new ways to perform meaningful actions, promising to the user an active exploration. The keyboard and the console, part of the older computer 'interactives' represent the passive mechanical channel between the computer and the user (Paterson 2007) as well as the object itself being a barrier to the 'immediacy' with the information. The ways we move, sweep, shift, tap the touch sensitive interfaces within the museum spaces challenge in many ways the familiar interaction with the world around us. This challenge provokes the audience's relationship with comprehending and getting information, and invites them to a sensory enhancement.

The Roman era galleries of the Museum of London, that were built and developed first, encapsulate in the exhibition space small computer screens accompanied by a keyboard, controllers or consolers aimed to one person's view. Computer interactives tend to be static, the size of your average computer monitor, and navigated by a sole individual (indeed a sole finger) providing information on a number of different levels. They tend to be characterised by their kiosk appearance and (necessarily) dark surroundings. (Kidd et al. 2011). The manipulation of these computer interactives seems to be more tedious for the audience, especially when familiar with new technologies.
The modern galleries at the Museum of London, a project developed in the last five years, have certainly taken new avenues drifting from the 'computer interactives' to touch and multi-touch interfaces. The two types of so-called interactives differ in many ways. Touch interfaces that allow multiple users (Figure 1) may house similar kinds of information, but are differentiated by the mode and aesthetics of their presentation. The interface tends to be a flat, horizontal tablet screen that can be accessed (and read) by a variety of people at any given time. There is a range of interesting aspects to be discussed by this move including the screen manipulated by finger and hand touch as well as the aspect of including more than one person within the same screen enabling social interaction.

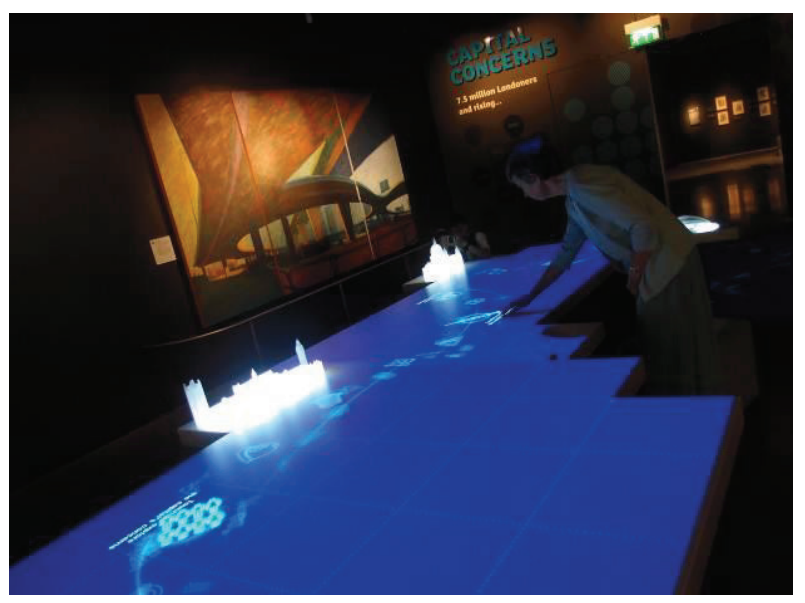

Figure 1: Capital Concerns, Museum of London

\section{RESEARCH}

This section will focus on the element of touch sensitive interfaces, aiming to consider and investigate the role of touch in the audience museum experience through digital 'interactives'. How touching is affecting these interactive experiences within the museum space? It is argued that the new and digital technologies afford new possibilities of experience, shaping the way we learn, apprehend and live the world around us, offering a multi-sensory understanding.

The purpose of the research was to try and establish any shift in terms of the (visible) nature of experience and interaction in the transition from kiosk-style computer interactives to the increased use of touch-screen interfaces, and to begin to reflect on terminology around 'interaction' more generally. 


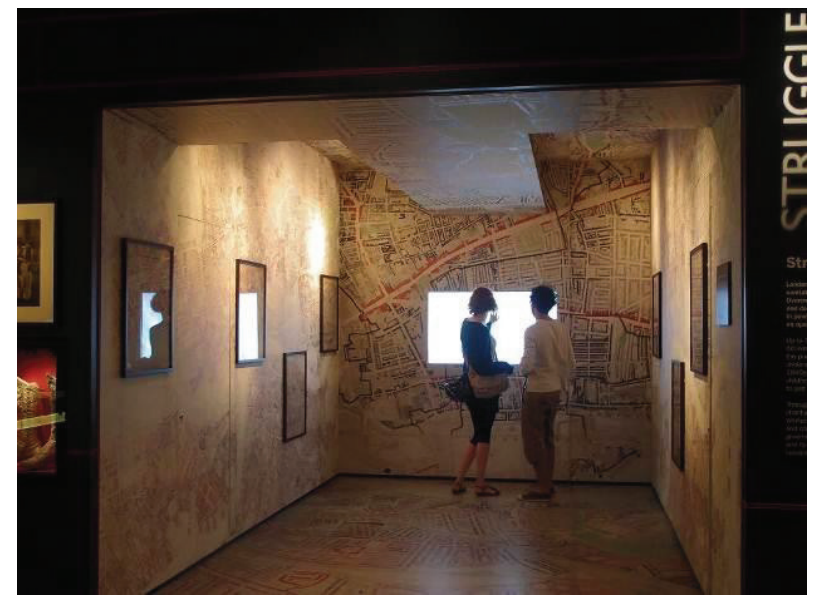

Figure 2: Museum of London

Previous studies used observations to look into the use of touch and multi-touch interfaces, to understand the conceptualization of 'social museum' and the relationships between collective and individual engagement; the body and experience; the group and knowledge construction (Kidd et al. 2011). The methodology used in my study comes from ethnomethodological approach using interviews by visitors and staff and observations (notes, sound recording).

\subsection{Sources of data}

The case study at the Museum of London included research at five touch-screen interfaces and the finding are extracted from:

- 53 interviews, 19 males and 34 females

- Approximately 12 hours of audio recording

\subsection{Headline findings}

Note: Further work on findings and analysis of the data will take place over the coming months. The following presents only a brief overview of those findings, which are emerging as 'headlines'.

As anticipated, elements of interactivity and interaction were observed at touch-screen interfaces. This includes discussion within the group, but also, discussion beyond the group (i.e. with other visitors in the gallery).

"I like the idea of touch screens, I think it's really exciting and new and...i like that museums embrace new technology em...working in the museum myself so I'm quite familiar with..with that kind of technology"

"I especially like the sort of an interactive map because I live in London so it's interesting to sort of touch the different, to see if I can see it on the map and then it'll show me if I was right or not.. I think people like to see..you know just to guess at things and then to see if they've got them right ...makes it sort of more like a game"

"It is "social interaction as well".., "with the children there is lots of interaction (laughter)"

"This one is very interesting, touch-screen we were just playing with it, kind of..you're learning about people's lives you know..cause you hear so much about the kings and queens or you know very well and this one is about common people, probably had lives that we don't know unfortunately"

As noted in the quotes above, interactivity is a new becoming (Deleuze \& Guattari 1987) in museum studies. It is a main concept in social studies and the museum-form of interactivity involves new technologies, affect, sociality, activity, engagement, technology as a tool, and pedagogical activity (Bannon \& Hall 2005) for adults as well for children. We can take this a little further. The perception expresses the obvious way in which the body and mind need each other to express the world (Murphy 2002). In terms of perception and interactives in the museum, this consists not only seeing. It is a space of affect, a "smooth space" (Deleuze \& Guattari 1987: 479), occupied by intensities, movements, becomings. It is haptic, rather than optical perception.

\begin{abstract}
"when you are in the museums where is a lot of texts and is a lot of things to look at is kind of fun when you have something that you can actually interact with cause with a lot of stuff you can't touch, you just look at..with this you can actually physically kind be involved so it's a lot of ..it's very.."
\end{abstract}

"And you touch the picture and you can see the presence and the future and you can give your opinion. I like that one very much, I think that's a good one." (intensity)

"'I like you can touch it and you can go over the map by touching it...that"

"You try to, you listen something yeah... you watch something, you have more interaction and those kinds of..am..movements and this activity is very helpful to understand, to well take some touch also but I believe that should be it. Very interesting'

"yeah move on and I think the first feeling is just that once you touch everything..there s a car, let me touch the car and understand"

The relationship between the interactivity and touching also emerged as an interesting one. In Capital Concern's interface for example, visitors enjoyed the system, which allowed them to test knowledge and to learn new things. It is a kind of social engagement, not just with the physical 
beings around them, but with the 'virtual' visitors who have been before them.

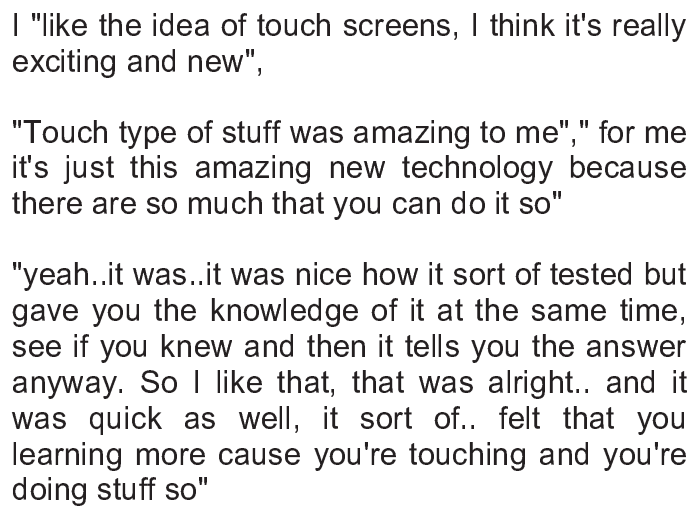

"Touch type of stuff was amazing to me"," for me it's just this amazing new technology because there are so much that you can do it so"

"yeah.. it was. .it was nice how it sort of tested but gave you the knowledge of it at the same time, see if you knew and then it tells you the answer anyway. So I like that, that was alright.. and it was quick as well, it sort of.. felt that you learning more cause you're touching and you're doing stuff so"

In terms of physical touching when using interfaces we noticed that the patterns varied. Some of the groups touched the screen together, discussing the information co-operatively and finding a route through it, but mostly it tended to be one member of the group touching the interface and 'leading' the enquiry.

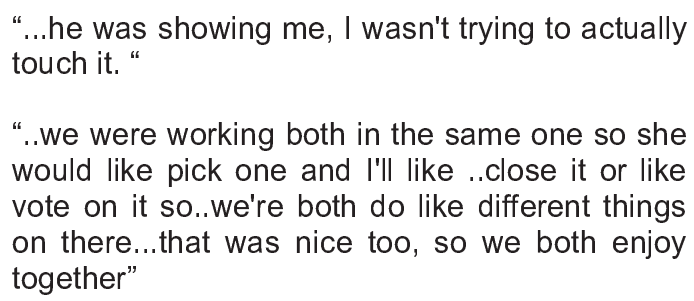

"..we were working both in the same one so she would like pick one and I'll like ..close it or like vote on it so..we're both do like different things on there...that was nice too, so we both enjoy together"

"yeah, she was pressing the button I was looking at results.."

Some others were not able to remember actions of touching and did not feel comfortable when other people were occupying the interactive and hung back to wait or walked on.

\footnotetext{
"You need to be able to actually see stuff and actually touch things"

"I would like tap the answer really hard but it had to be a light soft touch...a"

"yeah, but I was hard because it says just touch the sc...to answer the questions, it didn't necessarily point explain slide the touch"

"yes..yeah..but it was still hard..all it said was touch here to select and it doesn't"
}

\section{DISCUSSION}

Certainly, the over-reliance and excitement of cultural institutions such as museums on embracing interactive computer systems within their physical environment is an ongoing discussion. It is not arguable, that further investigation is required to deploy the audience experience when interacting with digital interactives within the specific context. Phenomenology can provide a valid methodological approach for researching museum audiences' experiences (Dudley 2010), making sense of the audiences encounters with digital interactive exhibits and exhibitions as experienced through touch, body and movements.

Overall, what emerges as important in the interfaces and interactivity is the experience of haptic, optical and affect, which certainly needs to be taken into account. When the interactive exhibits are properly designed and have added the aspect of touching as emotional experience, they allow users sharing learning experiences in the museum, bringing history and meaning of the collection to life. This excitement on the touch screen interfaces becomes obvious through the responses on the specific research as well as the acceleration on the use of touch-sensitive technologies in our everyday life. Hein (2001) argues that we love the simple, clean surfaces that computer generate, the way that the complexity and ambiguity of them is reduced clothed under colour and geometrical shapes and our affair with them is deeper than aesthetic fascination and deeper of play with senses.

There is space in the field of multi touch interfaces within the museums to conduct an inter-disciplinary research which will integrates understandings of the concepts of interactivity. The areas of interest that we recognise as needed to be explored involve, affect, body, social interaction and learning of the visitors in the museum space which facilitate the multi touch interface, the private and collaborative participation of the user and last but not least the notion of interactivity and interaction pattern of the visitors in the space.

\section{REFERENCES}

Anderson, G. (2004) Reinventing the Museum: Historical and Contemporary Perspectives on the Paradigm Shift, Walnut Creek, Calif. AltaMira Press.

Bumpus, H. C. (1907) A Contribution to the Discussion on Museum Cases, MJ, 6 (9) (March 1907): 299.

Classen, C, and D. Howes (2006) The Museum as Sensescape: Western Sensibilities and Indigenous Artifacts. In Edwards, E, C. Gosden \& R. B. Philip (Eds.), Sensible Objects. Colonialism, Museums and Material Culture (pp. 199-222). Oxford and New York, Berg.

Cranny-Francis, A. (2008) Touching Skin: Embodiment and the Senses in the Work of Ron Mueck. In Nicole Anderson \& Katrina Schlunke 
(eds.), Cultural Theory in Everyday Practice. Oxford University Press.

Deleuze, G., and Guattari, F. (1987) A thousand plateaus. (R. Hurley, M. Seem and H. R. Lane, Trans.). Minneapolis, MN: University of Minnesota Press.

Dourish, P. (2001) Where the Action Is: The Foundations of Embodied Interaction. Cambridge, Massachusetts: The MIT press.

Dreyfus, H.L. (1992) What computers still can't do: a critique of artificial reason. MIT Press Cambridge, MA, USA.

Dudley, S. (ed.). (2010) Museum Materialities: Objects, Engagements, Interpretations. London \& New York: Routledge.

Elo, M. (2012) Digital finger: beyond phenomenological figures of touch. Journal of Aesthetics \& Culture, 4, 1-12.

Gordon, D. (2001) Postmodernism and the Enlightenment, London: Routledge.

Griffiths, A. (1999) Media Technology and Museum Display: A Century of Accommodation and Conflict. Presentation at the MIT Communications Forum, December 19, 1999.

Hein, G. (1998) Learning in the Museum. London: Routledge

Hein, G. (2000) The museum in transition: A philosophical perspective. Washington and London: Smithsonian Institution Press.

Lakoff, G., and Johnson, M. (1999) Philosophy in the Flesh: The Embodied Mind and its Challenge to Western Thought. New York: Basic Books.

Lister, M., Dovey, J. Giddings, S. Grant, I., and Kelly, K. (2003) New Media: A Critical Introduction. London: Routledge.
Lord, B. (2006) Foucault's Museum: difference, representation, and genealogy, Museum and Society, 4 (1), 1-14.

Kidd J., Ntalla I., and Lyons W. (2011) Sensing the Social Museum. International Conference 'Re thinking technologies in museums 2011 - Emerging Experiences', Limerick, Ireland. (Conference presentation/Publication).

Merleau-Ponty, M. (1993) Indirect Language and the Voices of Silence, trans. R.C. McCleary, in G.A. Johnson (ed.), The Merleau-Ponty Aesthetics Reader, 76-120, Evanston: Northwestern University Press.

Munster, A. (2006) Materializing New Media: Embodiment in Information Aesthetics. Hanover: Dartmouth.

Ntalla I. (2012) Interactivity and audience experience in the modern museum; discussing findings from case study on the 'High Arctic' immersive installation, National Maritime Museum, London. Transformative Museum Conference, Roskilde University, Denmark, 2012. (Conference publication.)

Paterson, M. (2007) The Senses of Touch. Oxford \& New York: Berg.

Vygotsky, L. (1978) Mind and society: the development of higher mental processes. Cambridge, MA, Harvard University Press.

Vom Lehn, D., and Heath, C. (2005) Accounting for New Technology in Museums. International Journal of Arts Management, 7:11-21. 